\title{
The Differential Phase Pattern of the CSU CHILL Radar Antenna
}

\author{
A. Mudukutore, V. Chandrasekar, and E. A. Mueller \\ Colorado State University, Fort Collins, Colorado \\ 31 May 1994 and 27 February 1995
}

\begin{abstract}
The measurement of the differential propagation phase and copolar correlation coefficient are affected by the differential phase pattern of the antenna system when operating in an alternate horizontal and vertical transmitting scheme. Direct phase pattern measurements of a large dish such as that of the CSU CHILL is difficult because of the need to obtain phase reference. A simple technique is devised to measure the differential phase pattern of the CSU CHILL, antenna system. The measurements are subsequently used in the evaluation of the antenna imposed limit on the copolar correlation coefficient.
\end{abstract}

\section{Introduction}

Antenna characteristics play an important role in the accuracy of polarimetric radar measurements. The antenna differential phase $\Delta \Phi_{\mathrm{ANT}}$, defined as the difference in the $H$ and $V$ phase patterns, should be constant within the main lobe for an ideal antenna. However, imperfections in the reflector geometry, improper positioning of the feed horn, or primary pattern effects of the struts can cause differences between the horizontal and vertical phase patterns within the main lobe, which, when operating in an alternate horizontal and vertical transmitting scheme, limits the accuracy in the measurements of the differential propagation phase shift $\Phi_{D P}$ and the magnitude of the zero-lag copolar correlation coefficient between horizontal and vertical polarized received signals $\rho_{H V}(0)$ (Xiao et al. 1993). This paper presents a technique to obtain the differential phase pattern $\left(\Delta \Phi_{\text {ANT }}\right)$ for the CSU CHILL radar antenna and evaluate its impact on the measurement of $\rho_{H V}(0)$.

\section{CSU CHILL antenna system}

The CSU CHILL uses a fully steerable, primary focus-fed paraboloidal reflector antenna housed in a 22 $m$-diameter inflated radome. The diameter of the reflector is $8.5 \mathrm{~m}$ with three struts and a double waveguide running along the $\phi= \pm 45^{\circ}$ planes. The $H$ and $V$ ports from the polarization-switch feed into a Potter-type dual-polarization horn located at the focal point of the

Corresponding author address: A. Mudukutore, Department of Electrical Engineering, Colorado State University, Fort Collins, $\mathrm{CO}$ 80523.

E-mail: am511113@longs.lance.colostate.edu reflector. The half-power beamwidth of the antenna is approximately $0.9^{\circ}$, and the sidelobes are down greater than $27 \mathrm{~dB}$ with respect to the main-lobe peak.

\section{Data collection}

The procedure that was used to measure the differential phase antenna patterns is conceptually straightforward, but in practice, many details have to be considered for maintaining the data quality. Direct phase pattern measurements of a large dish such as that of the CSU CHILL are difficult because of the need to obtain a phase reference. However, since we are interested only in the differential phase pattern of the antenna between horizontal and vertical polarizations, a novel scheme was devised to achieve this.

The test setup consisted of a standard gain pyramidal horn-antenna as the test source, located on top of a tower about $4.5 \mathrm{~km}$ west of the radar at an elevation angle of $0.5^{\circ}$. The far field of the CSU CHILL antenna $\left(2 D^{2} / \lambda\right)$ is $1400 \mathrm{~m}$, and therefore, the test source was well into the far field. The transmit horn was connected to a CW (continuous wave) source (Hewlett-Packard synthesizer), and the transmit frequency was set to be close to that of the CSU CHILL transmitter (a few kilohertz difference). The polarization state of the transmit horn was set to be $45^{\circ}$ linear polarization with respect to the horizontal. The radar was operated in an $H / V$ switched mode, switching between horizontal and vertical polarization states every millisecond. The antenna was scanned over an azimuth and elevation grid about the bore sight at a slow scan rate collecting several samples at each position (64 or 128 samples). Measuring the upper half of the pattern required that we rotate the antenna $180^{\circ}$ in azimuth and flip it by $180^{\circ}$ in elevation and scan in decreasing elevation steps. 
This procedure gave a $0.5^{\circ}$ overlap region between the top and bottom halves of the pattern.

\section{Measurement principle}

The transmit horn and the CSU CHILL radar (the receive system) have independent sources. It may appear as a result of independent oscillators that meaningful phase measurements are not possible. However, differential phase measurements can be achieved so long as the oscillators at the radar and the test source remain coherent over two pulse repetition times.

The phase of the horizontal component at time ( $2 n$ +1) $T_{s}$ can be expressed as

$$
\begin{aligned}
\Phi^{[H]}(2 n+1)=\Phi_{\mathrm{TX}}^{[H]} & +\Phi_{\text {air }}^{[H]}(2 n+1) \\
& +\Phi_{\mathrm{WG}}^{[H]}+\Phi_{\mathrm{SW}}^{[H]}+\Phi_{\mathrm{ANT}}^{[H]},
\end{aligned}
$$

where

- $\Phi_{\mathrm{TX}}^{[H]}$ is the phase contribution due to the transmitter for the horizontal component,

- $\Phi_{\text {air }}^{[H]}(2 n+1)$ is the phase shift through air at time $(2 n+1) T_{s}$

- $\Phi_{\mathrm{WG}}^{[H]}$ is the phase shift due to the electric length of the waveguide in the $H$ polarization path,

- $\Phi_{\mathrm{SW}}^{[H]}$ is the phase shift induced by the switchable circulator, and

- $\Phi_{\mathrm{ANT}}^{[H]}$ is the phase shift due to the antenna pattern.

Similarly, the phase of the vertical component at time (2n) $T_{s}$ can be expressed as

$$
\begin{aligned}
\Phi^{[V]}(2 n)= & \Phi_{\mathrm{TX}}^{[V]}+\Phi_{\text {air }}^{[V]}(2 n)+\Phi_{\mathrm{WG}}^{[V]} \\
& +\Phi_{\mathrm{SW}}^{[V]}+\Phi_{\mathrm{ANT}}^{[V]}-2 \pi\left(f_{1}-f_{2}\right) T_{s},
\end{aligned}
$$

where the individual contributions are the same as before but at the vertical polarization state. Also, $2 \pi\left(f_{1}\right.$ $\left.-f_{2}\right) T_{s}$ is the phase contribution due to the difference in the test source transmitter frequency $\left(f_{1}\right)$ and the frequency of the coherent oscillator in the radar $\left(f_{2}\right)$. Therefore,

$$
\begin{aligned}
& \Phi^{[H]}(2 n+1)-\Phi^{[V]}(2 n)=\Delta \Phi_{\mathrm{air}}(2 n, 2 n+1) \\
& \quad+\Delta \Phi_{\mathrm{WG}}+\Delta \Phi_{\mathrm{SW}}+\Delta \Phi_{\mathrm{ANT}}+2 \pi\left(f_{1}-f_{2}\right) T_{s}
\end{aligned}
$$

where

- $\Phi_{\mathrm{TX}}^{[H]}=\Phi_{\mathrm{TX}}^{[V]}$,

- $\Delta \Phi_{\text {air }}(2 n, 2 n+1)$ is the fluctuation in the refractive index along the path in the time interval $(2 n, 2 n$ $+1)$ and it is assumed $\Phi_{\text {air }}^{[H]}(2 n)=\Phi_{\text {air }}^{[V]}(2 n)$ [even if $\Phi_{\text {air }}^{[H]}(2 n)$ is not nearly the same as $\Phi_{\text {air }}^{[V]}(2 n)$, it is sufficient for our purposes that the difference between them stays the same over the measurement time],

- $\Delta \Phi_{\mathrm{WG}}=\Phi_{\mathrm{WG}}^{[H]}-\Phi_{\mathrm{WG}}^{[V]}$,

- $\Delta \Phi_{\mathrm{SW}}=\Phi_{\mathrm{SW}}^{[H]}-\Phi_{\mathrm{SW}}^{[V]}$, and

- $\Delta \Phi_{\mathrm{ANT}}=\Phi_{\mathrm{ANT}}^{[H]}-\Phi_{\mathrm{ANT}}^{[V]}$ is the differential phase contribution due to the antenna pattern.
Similarly,

$$
\begin{aligned}
& \Phi^{[V]}(2 n)-\Phi^{[H]}(2 n-1)=\Delta \Phi_{\mathrm{air}}(2 n-1,2 n) \\
& \quad-\Delta \Phi_{\mathrm{WG}}-\Delta \Phi_{\mathrm{SW}}-\Delta \Phi_{\mathrm{ANT}}+2 \pi\left(f_{1}-f_{2}\right) T_{s} .
\end{aligned}
$$

The sum of (3) and (4) yields the average phase shift $\Phi_{\text {avg }}$ to be

$$
\begin{aligned}
\Phi_{\mathrm{avg}} & =\frac{\Phi^{[H]}(2 n+1)+\Phi^{[H]}(2 n-1)}{2} \\
& =0.5 \Delta \Phi_{\mathrm{air}}(2 n-1,2 n+1)+2 \pi\left(f_{1}-f_{2}\right) T_{s} .
\end{aligned}
$$

A necessary condition for accurate measurement of the differential phase pattern of the antenna is that $\Phi_{\text {avg }}$ should be nearly constant as a function of the sampling time. Figure 1 shows ' $\Phi_{\text {avg }}$ (in degrees) plotted as a function of sample-time index $n$ (sample spacing $T_{s}$ $=1 \mathrm{~ms}$ ), with the radar antenna pointing directly at the test source. It can be seen from the figure that there are no increasing or decreasing trends in the average phase shift and only small fluctuations about the mean value exist (standard deviation is $0.67^{\circ}$ ) due to changes in the refractive index along the path. These fluctuations are small and do not cause significant errors in the $\Delta \Phi_{\text {ANT }}$ measurements. From the ensemble average of the difference in phase in (3) and (4), we have

$$
\begin{aligned}
\Delta \Phi_{\mathrm{ANT}}+\Delta \Phi_{\mathrm{SW}}+\Delta \Phi_{\mathrm{WG}} \\
\\
=\frac{\left\langle\Phi^{[H]}(2 n+1)-\Phi^{[/]}(2 n)\right\rangle}{-\left\langle\Phi^{[/]}(2 n)-\Phi^{[H]}(2 n-1)\right\rangle} \\
2
\end{aligned}
$$

where we assume $\left\langle\Delta \Phi_{\text {air }}\right\rangle=0$.

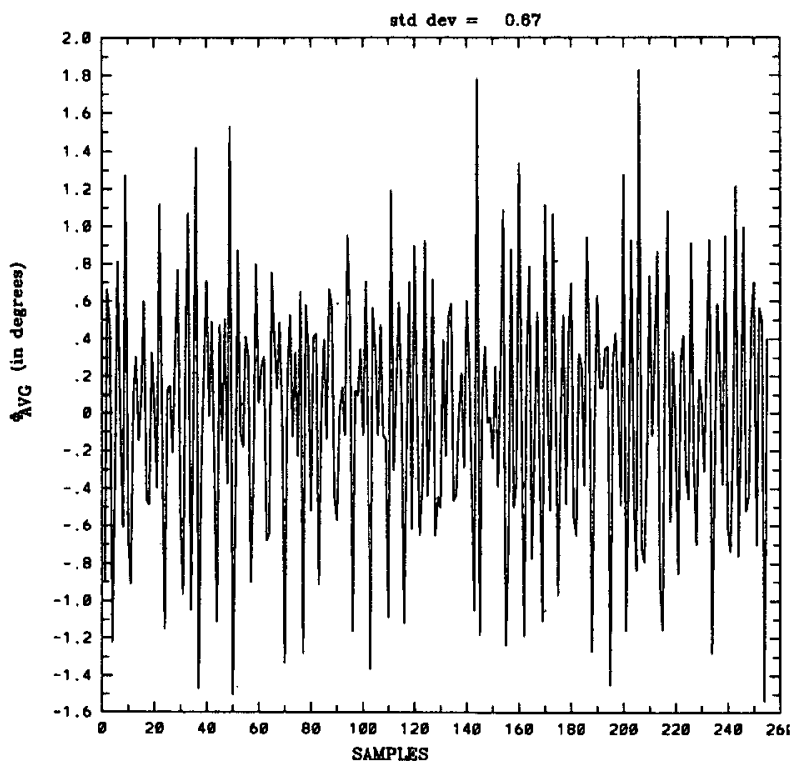

FlG. 1. Plot of $\Phi_{\text {avg }}$ with time. 
As the radar antenna is scanned about the test source, $\Delta \Phi_{\mathrm{SW}}$ and $\Delta \Phi_{\mathrm{WG}}$ remain constant and therefore can be subtracted out from (6). The next section shows test data analyzed with the above model.

\section{Results}

Figure 2 shows $\Delta \Phi_{\mathrm{ANT}}$, the mean differential phase shift across the main beam and sidelobes, plotted as a function of azimuth angle at various fixed elevation angles. Note that the azimuth and elevation angles used in this figure are with respect to the azimuth and elevation of the source. It can be observed from the figure that there is a small change in the differential phase across the main lobe and abrupt changes at the first sidelobes. Using the geometrical theory of diffraction (GTD) technique (Chang and Rudduck 1982), the antenna $\Delta \Phi_{\text {ANT }}$ pattern was evaluated numerically and is shown in Fig. 3. The GTD technique takes into account the effects of blockage due to the struts and feedhorn. The Potter-type dual-polarization horn is numerically modeled. The differential phase patterns obtained from measurement and numerical computation (GTD technique) are in general agreement. It can be seen from the pattern that the $\Phi_{\mathrm{DP}}$ estimates will be noisy when the antenna is illuminating stronger targets through the sidelobes due to the extensive fluctuations of $\Delta \Phi_{\text {ANT }}$ at the sidelobes.

The cross-correlation coefficient measurement upperbound limit $\left(\rho_{A}\right)$ due to the differential phase pattern of the antenna for a beam filled uniformly with isotropic scatterers, neglecting second-order cross-polar terms, is defined as (Liu et al. 1994)

$$
\rho_{A}=\frac{\left|\int f_{H H} f_{V V} e^{j 2 \Delta \Phi_{A N T}} d \Omega\right|}{\left[\int\left|f_{H H}\right|^{2} d \Omega \int\left|f_{V V}\right|^{2} d \Omega\right]^{1 / 2}},
$$

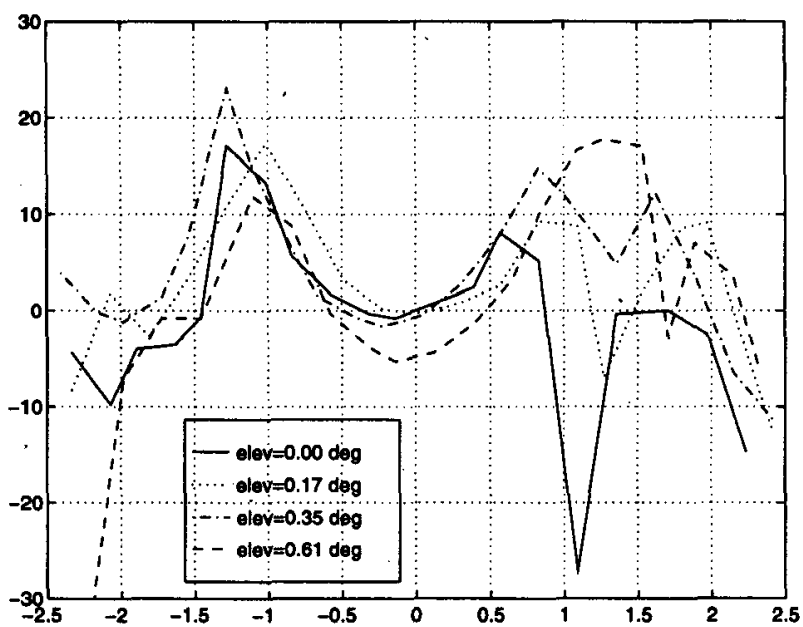

FIG. 2. Plot of $\Delta \Phi_{\mathrm{ANT}}$ vs azimuth at different fixed elevations.

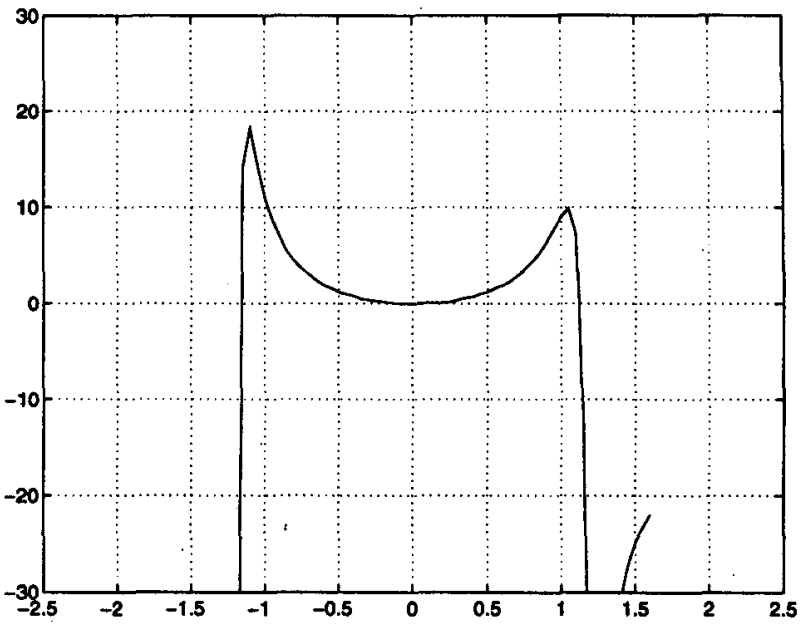

FIG. 3. Plot of numerically obtained $\Delta \Phi_{\text {ANT }}$ vs azimuth.

where $f_{H H}$ and $f_{V V}$ are the conventional copolar oneway power patterns in the horizontal and vertical polarization states, respectively. Two-dimensional complex integration was performed over a grid of size $5^{\circ}$ $\times 5^{\circ}$ about the bore site to compute the $\rho_{A}$ of the antenna. The results from the integration yielded an upper bound on $\rho_{H V}(0)$ measurements given by $\hat{\rho}_{A}=0.990$.

\section{Summary and conclusions}

We have devised a simple scheme to measure the differential phase pattern of the CSU CHILL radar. The measurements show good agreement with the numerical calculations obtained using the geometric theory of diffraction technique. Based on the measured differential phase pattern of the antenna, the limit on cross-correlation coefficient measurement was estimated to be 0.990 . In contrast, Illingworth and Caylor (1991) have reported a measured upper bound for $\rho_{H V}(0)$ of 0.997 to 0.998 using the RAL Chilbolton radar looking at light rainfall. Also, both the National Severe Storm Laboratory Cimarron and the National Center for Atmospheric Research CP-2 radars appear to have a measured upper limit for $\rho_{H V}(0)$ of 0.985 to 0.990 (Balakrishnan and Zrnić 1990; Liu et al. 1993). Based on the results presented in this paper, it appears that these bounds are primarily due to antenna imperfections.

Acknowledgments. The authors acknowledge helpful discussions with Prof. Bringi of CSU. The authors also acknowledge the careful review and helpful suggestions of Dr. Doviak of NSSL. This research was supported by the National Science Foundation via Grant ATM9019596.

\section{REFERENCES}

Balakrishnan, N., and D. S. Zrnić, 1990: Use of polarization to characterize precipitation and discriminate large hail. J. Atmos. Sci., 47, $1525-1540$. 
Chang, Y.C., and R. C. Rudduck, 1982: Numerical Electromagnetic Code-Reflector Antenna Code. Tech. Rep. 712242-17, Ohio State University.

Illingworth, J. A., and I. J. Caylor, 1991: Co-polar correlation measurements of precipitation. Preprints, 25th Int. Conf. on Radar Meteorology, Paris, France, Amer. Meteor. Soc., 650-653.

Liu, L., V. N. Bringi, I. J. Caylor, and V. Chandrasekar, 1993: Intercomparison of multi-parameter radar signatures from Florida storms. Preprints, 26th Int. Conf. on Radar Meteorology, Norman, OK, Amer. Meteor. Soc., 733-735.
,-- V. Chandrasekar, E. A. Mueller, and A. Mudukutore, 1994: Analysis of the copolar correlation coefficient between horizontal and vertical polarizations. J. Atmos. Oceanic Technol., 11, 950963.

Xiao, R., V. N. Bringi, D. Garbrick, E. A. Mueller, and S. A. Rutledge, 1993: Copolar and cross-polar pattern measurement of the CSU CHILL antenna. Preprints, 26th Int. Conf. on Radar Meteorology, Norman, OK, Amer. Meteor. Soc., $363-365$ 